\title{
Composition and Antibacterial Activity of the Lipophilic Fraction of Honeybee Pollen from Native Species of Montesinho Natural Park
}

\author{
${ }^{1,2}$ Sandra I.T.R. Barbosa, ${ }^{1}$ Armando J.D. Silvestre, \\ ${ }^{1}$ Mário M.Q. Simões and ${ }^{2}$ Maria L.M.F. Estevinho \\ ${ }^{1}$ Department of Chemistry, University of Aveiro, 3810-193 Aveiro, Portugal \\ ${ }^{2}$ Department of Biology, Escola Superior Agrária, \\ Instituto Politécnico de Bragança, 5300 Bragança, Portugal
}

\begin{abstract}
The lipophilic composition of honeybee pollen from Cistus ladanifer, Castanea sativa and Rubus sp. was analysed by GC-MS. The extracts are mainly composed by saturated and unsaturated fatty acids, sterols, long chain aliphatic alcohols, alkanes and alkenes. The profiles of chemical composition allow the chemical differentiation of pollen species. The biological activity of the lipophilic fractions against several Gram positive bacteria was demonstrated and related to the resistance of beehives to certain diseases when feed with such pollens.
\end{abstract}

Key words: Lipophilic extractive, honeybee pollen, Cistus ladanifer, Castanea sativa, Rubus sp., GC-MS analysis, antibacterial activity

\section{Introduction}

Honeybee pollen is one of the most prominent beehive's products. Due to its therapeutic properties and nutritional value, it has long been used not only in folk medicine but also as a dietary supplement.

Bee pollen is promoted as a dietary supplement with a wide range of nutritional and therapeutic properties. In fact, a wide number of promising properties of this natural product have been reported in the literature: for example, its protective action against side effects of radiotherapy in cancer patients (Wenning, 2003), its use in the treatment of allergic rhinitis (Boye et al., 1990; Staff et al., 1990) and its activity as an hepatoprotector (Juzwiak et al., 1992) along to its antiatherogenic effect (Ji et al., 1989; Zhao et al., 1990); in the treatment of some cases of benign prostatitis (Denis, 1966; Askupmar, 1967; Samochowiec et al., 1992; Rugendorff et al., 1993) and in the desensitization of children allergic to pollen (Wortmann, 1981; Krell, 1996). The regular consumption of honeybee pollen contributes to reduce the seric levels of triglycerides and cholesterol (Wójcicki et al., 1983). On the other hand, some studies reveal that the pollen's extract reduces not only the lipidic levels of the serum, but also the intensity of the atherosclerotic plaque (Wójcicki et al., 1986), contributing to a lower platelet aggregation, both in vitro and in vivo (Wójcicki and Samochowiec, 1984; Kosmider et al., 1983).

Despite the earlier mentioned properties, there is still some lack of knowledge on the relationship between the chemical composition of honeybee pollen and the reported biological properties. On the other hand, the chemical composition of honeybee pollen composition is variable and strongly dependent on the edafoclimatic conditions and on the botanical biodiversity present in the region where the beehives are located.

Corresponding Author: Armando J.D. Silvestre, Associate Professor, Department of Chemistry, University of Aveiro, 3810-193 Aveiro, Portugal Tel: 351234370711 Fax: 351234370084 
Pollen is a natural source of proteins, fats, minerals and vitamins, which are necessary elements for the normal development of a bee colony (grubs and bees) and, likewise, are important for human nutrition (Cobo, 1984).

Concerning the lipophilic fraction of honeybee pollen extractive, published results demonstrate that they are mainly composed by fatty acids and hydrocarbons (Bianchi et al., 1990; Bonvehí and Jordà, 1997; Saa-Otero et al., 2000). However, in most studies the number of identified compounds is quite limited due to the detection/identification techniques used (mainly based on GC-FID and comparison with standards). A special attention has been devoted to this fraction because fatty acids play an important role in honeybee nutrition as they do for humans and additionally, these compounds also play another extremely important role: their antimicrobial activity. For example, linoleic and linolenic acids are bacterial growth inhibitors. In humans, for example, tests have showed that linoleic acid inhibited the etiologic agent of chronic gastritis and peptic ulcer, Helicobacter pylori (Petschow et al., 1996).

In an earlier work it has been observed that, in beehives with an incidence of the pathogenic fungus Ascophaera apis, there was a decrease in the incidence of European foulbrood caused by Melissococcus pluton and it has been demonstrated that this inhibition was caused by the presence of linoleic acid produced by such fungus (Feldlaufer et al., 1993a). It was also demonstrated that linoleic acid inhibited the formation of American foulbrood spores caused by Paenibacillus larvae larvae (Feldlaufer et al., 1993b), a bacteria with the capability of forming spores which remain viable for long periods and survive to adverse conditions.

Since the studied pollen samples were shown to have considerably high amounts of fatty acids with high antimicrobial activity, this might indicate that we could be in the presence of pollens with biocide action, just like canola (Brassica napus) pollen (Manning, 2001).

For the last 30 years, thanks to the drive for a more natural diet in Europe and especially in France, a development of apiculture to produce pollen has been observed, due to its nutritional and medicinal properties (Liebelt, 1994). As a result, regions where traditionally only honey was produced, became potential producers of high quality bee-collected pollen. This is the case of the region of Montesinho Natural Park (MNP), the biggest Portuguese region with origin denomination in honey production.

Although many plant species, relevant as honeybee pollen providers, have been intensively studied, to our knowledge the chemical composition of the pollen of Cistus ladanifer, Castanea sativa and Rubus sp., the main pollen sources in MNP, a natural park in the north of Portugal, has not been fully studied yet.

Within the scope of a project aiming to study beehives' products from MNP, in this research we report the study of the chemical composition of the lipophilic fraction of honeybee pollen, namely Cistus ladanifer, Castanea sativa and Rubus sp., in the period investigated, from May to July 2002. The lipophilic extracts were also submitted to antibacterial activity tests against three Gram positive bacteria namely Bacillus subtilis CECT 498, Bacillus cereus CECT 148 and Paenibacillus larvae and two Gram negative bacteria, namely Escherichia coli $\mathrm{CECT} 101$ and Pseudomonas aeruginosa CECT 108 , envisioning beehive's sanitary contributions.

\section{Materials and Methods}

Samples

Bee pollen was collected from two distinct areas in Montesinho Natural Park during the flowering months (May to July 2002). Pollen pellets were kept in a pollen trap for no more than $24 \mathrm{~h}$. The collected samples were lyophilised, colorimetricaly separated and identified by palinologic analysis according to the Erdtman (1969) method. 


\section{Extraction}

Each sample $(50 \mathrm{~g})$ of finely grounded pollen from predominant species, i.e., Cistus ladanifer, Castanea sativa, Rubus sp., were Soxhlet extracted with $n$-hexane for $12 \mathrm{~h}$. The solvent was evaporated to dryness and the extracts were weighed. The results were expressed in mg compound/g dry pollen.

\section{Alkaline Hydrolysis}

In order to detect the presence of fatty acids, alcohols and sterols in the esterified forms, $20 \mathrm{mg}$ of each extract were dissolved in $10 \mathrm{~mL}$ of $1 \mathrm{M} \mathrm{KOH}$ in $10 \%$ aqueous methanol. The mixtures were heated at $100^{\circ} \mathrm{C}$, under nitrogen atmosphere, during $1 \mathrm{~h}$. The reaction mixtures were cooled, acidified with $1 \mathrm{M} \mathrm{HCl}$ to $\mathrm{pH} 2$ and then extracted three times with dichloromethane. The solvent was then evaporated to dryness.

\section{CG-MS Analysis}

The GC-MS analysis and quantification of the major lipophilic pollen extractive (before and after alkaline hydrolysis) were performed as described elsewhere (Freire et al., 2002). Nearly $20 \mathrm{mg}$ of each dried sample were trimethylsilylated and injected three times. GC-MS analyses were performed using a Trace Gas Chromatograph 2000 Series equipped with a Finnigan Trace MS mass spectrometer, using helium as the carrier gas $\left(35 \mathrm{~cm} \mathrm{~s}^{-1}\right)$, equipped with a DB-1 JW capillary column $(30 \mathrm{~m} \times 0.32 \mathrm{~mm}$ i.d., $0.25 \mu \mathrm{m}$ film thickness). The chromatographic conditions were as follows: initial temperature: $80^{\circ} \mathrm{C}$ for $5 \mathrm{~min}$; temperature rate: $4^{\circ} \mathrm{C}$ per min; final temperature: $285^{\circ} \mathrm{C}$ for $10 \mathrm{~min}$; injector temperature: $290^{\circ} \mathrm{C}$; transfer-line temperature: $290^{\circ} \mathrm{C}$; split ratio: $1: 100$.

For quantitative analysis a measured amount of internal standard (tetracosane) was added to each sample. The respective multiplication factors needed for quantification of the peak areas were calculated as an average of three GC-MS runs.

Compounds were identified, as TMS derivatives, by comparing their mass spectra with the GC-MS spectral library, with data from the literature and in some cases, by the injection of standards.

\section{Determination of Antibacterial Effect by Agar Well Diffusion Method}

Suspensions of three Gram positive bacteria namely Bacillus subtilis CECT 498, Bacillus cereus CECT 148 and Paenibacillus larvae and two Gram negative bacteria, namely Escherichia coli CECT 101 and Pseudomonas aeruginosa CECT 108, were prepared in order to contain approximately $10^{8} \mathrm{cfu} \mathrm{mL}^{-1}$ and the plates were inoculated. The lipophilic extracts were incorporated in a cavity (3 $\mathrm{mm}$ depth, $4 \mathrm{~mm}$ diameter) made in the center of the Petri plates $(9 \mathrm{~cm}$ diameter) with nutrient agar. The plates were incubated at $37^{\circ} \mathrm{C}$ for $24 \mathrm{~h}$ in duplicate. The inhibition zones were measured and expressed in $\mathrm{cm}$.

\section{Results and Discussion}

The pollen samples were identified on the basis of palynological analysis as monofloral Cistus ladanifer, Castanea sativa and Rubus sp. pollen.

\section{Analysis of the Lipophilic Extracts}

The extraction yields of the dichloromethane extract account for 1.1,2.0 and 3.6\% (on a dry basis) of C. sativa, Rubus sp. and C. ladanifer, respectively.

The composition of the lipophilic fraction of the studied extracts, as well as the abundances of individual components are shown in Table 1.

In general, the major lipophilic components of the three extracts are fatty acids (saturated and unsaturated), followed by alkanes, alkenes, sterols and alcohols (Fig. 1). The substantial increase in the amounts of extractives after alkaline hydrolysis and particularly in the amount of fatty acids, but also 


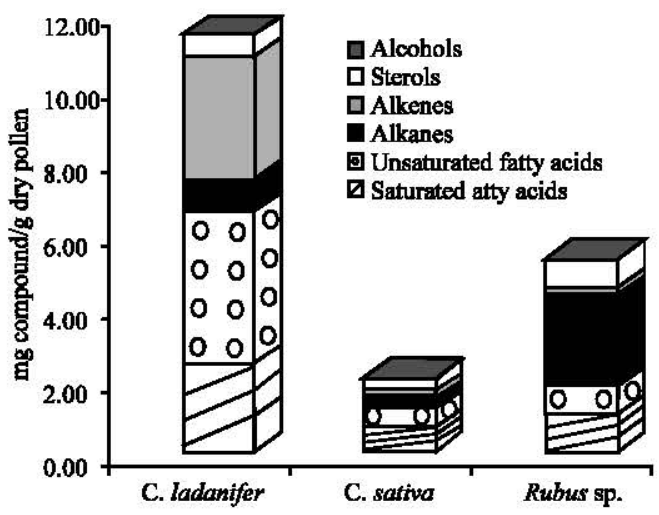

Fig. 1: Major families of compounds identified in the $n$-hexane extracts of bee pollen, before alkaline hydrolysis

Table 1: Chemical composition and abundances ( $\mathrm{mg} / \mathrm{g}$ of dry pollen) of the lipophilic fraction of C. ladanifer, C. sativa and Rubus sp. (BH: before alkaline hy drolysis; $\mathrm{AH}$ : after alkaline hydrolysis)

\begin{tabular}{|c|c|c|c|c|c|c|}
\hline & \multicolumn{2}{|c|}{ C. ladanifer } & \multicolumn{2}{|c|}{ C. sativa } & \multicolumn{2}{|c|}{ Rubus sp. } \\
\hline & $\mathrm{BH}$ & $\mathrm{AH}$ & $\mathrm{BH}$ & $\mathrm{AH}$ & $\mathrm{BH}$ & $\mathrm{AH}$ \\
\hline Octanoic acid & 0.0661 & 0.828 & 0.0273 & 0.0184 & 0.0118 & 0.0358 \\
\hline Nonanoic acid & 0.0732 & 0.0715 & 0.2095 & 0.1714 & 0.0271 & 0.0552 \\
\hline Decanoic acid & 0.2233 & 0.4992 & 0.0048 & 0.0122 & 0.0110 & 0.0242 \\
\hline Undecanoic acid & 0.0145 & 0.0318 & - & - & - & - \\
\hline Dodecenoic acid & $\operatorname{tr}$ & - & - & - & - & - \\
\hline Dodecanoic acid & 0.1076 & - & - & - & 0.0045 & 0.0172 \\
\hline Octanedioic acid & - & - & - & - & 0.0061 & 0.0106 \\
\hline Nonadioic acid & 0.0164 & 0.0470 & 0.0090 & 0.0098 & 0.0171 & 0.0236 \\
\hline Tetradecanoic acid & 0.0334 & 0.1119 & 0.0040 & 0.0109 & 0.0055 & 0.0457 \\
\hline Decanedioic acid & 0.0072 & 0.0949 & 0.0059 & $\operatorname{tr}$ & - & - \\
\hline Nonadecane & 0.0895 & 0.1206 & 0.0046 & - & - & - \\
\hline 1-Hexadecanol & - & - & - & - & 0.0055 & 0.1307 \\
\hline Hexadecenoic acid & 0.0103 & $\operatorname{tr}$ & - & - & - & - \\
\hline Hexadecanoic acid & 1.4038 & 2.7408 & 0.1171 & 0.2656 & 0.2462 & 1.0292 \\
\hline Heneicosene & 0.0142 & 0.1888 & - & - & - & - \\
\hline Heneicosane & 0.1674 & 0.2018 & 0.0081 & 0.0098 & 0.0514 & 0.0514 \\
\hline Z-9-Octadecen-1-ol & - & - & - & - & - & 0.0296 \\
\hline Heptadecanoic acid & 0.0075 & - & - & - & 0.0054 & 0.0370 \\
\hline 1-Octadecanol & - & - & - & - & 0.0119 & 0.0971 \\
\hline Linoleic acid & 2.5245 & 4.6843 & 0.1478 & 0.3808 & 0.0536 & 0.2863 \\
\hline Linolenic acid & 0.6988 & 1.6278 & - & - & 0.2758 & 1.3868 \\
\hline Oleic acid & 0.5231 & 1.4381 & 0.2195 & 0.8626 & 0.4838 & 1.7656 \\
\hline Octadecanoic acid & 0.1307 & 0.5625 & 0.0268 & 0.0630 & 0.0661 & 0.3520 \\
\hline Tricosene & 0.0261 & - & 0.0126 & 0.0141 & 0.0724 & 0.0945 \\
\hline 11-Octadecenoic acid & - & - & - & - & 0.0166 & 0.0357 \\
\hline Tricosane & 0.0848 & 0.1051 & 0.0948 & 0.0934 & 1.5908 & 1.6191 \\
\hline 1-Eicosanol & 0.0765 & 0.0635 & 0.0045 & 0.0144 & 0.0083 & 0.0642 \\
\hline 2-Hydroxy-9,12-octadienoic acid & 0.0594 & 0.1590 & - & - & - & - \\
\hline Eicosanoic acid & 0.0280 & 0.0658 & 0.0332 & 0.0477 & 0.1165 & 0.1461 \\
\hline Pentacosene & 0.0514 & 0.0604 & 0.0118 & 0.0112 & 0.0708 & 0.0686 \\
\hline Pentacosane & 0.1982 & 0.3234 & 0.0727 & 0.1001 & 0.8247 & 0.9307 \\
\hline Hexacosane & - & - & - & - & 0.0164 & $0 ., 0264$ \\
\hline Docosenoic acid & 0.2418 & 0.5295 & 0.0066 & 0.0274 & - & - \\
\hline Docosanoic acid & 0.0836 & 0.1138 & 0.0226 & 0.0296 & 0.4006 & 0.4254 \\
\hline Heptacosene & 0.0430 & 0.0278 & 0.0112 & 0.0104 & 0.0227 & 0.4254 \\
\hline Heptacosane & 0.1255 & 0.3954 & 0.0809 & 0.4606 & 0.2736 & - \\
\hline 1-Docosanol & - & - & 0.0103 & 0.0222 & - & - \\
\hline
\end{tabular}


Table 1: Continued

\begin{tabular}{|c|c|c|c|c|c|c|}
\hline & \multicolumn{2}{|c|}{ C. ladanifer } & \multicolumn{2}{|c|}{ C. sativa } & \multicolumn{2}{|c|}{ Rubus sp. } \\
\hline & $\mathrm{BH}$ & $\mathrm{AH}$ & $\mathrm{BH}$ & $\mathrm{AH}$ & $\mathrm{BH}$ & $\mathrm{AH}$ \\
\hline Linoleic acid 1-monoglyceride & 0.0308 & - & - & - & - & - \\
\hline Lanost-8-en-3-one & - & - & - & - & 0.0436 & - \\
\hline 1-Tetracosanol & - & - & - & - & 0.0089 & 0.0033 \\
\hline Tetracosenoic acid & 0.1115 & 0.2963 & 0.0171 & 0.0251 & - & - \\
\hline Tetracosanoic acid & 0.0434 & 0.0560 & 0.0318 & 0.0304 & 0.6574 & 1.0685 \\
\hline 2-Pentacosanol & - & - & 0.0863 & 0.2053 & - & - \\
\hline Nonacosene & 0.5012 & 0.6662 & - & - & - & - \\
\hline Nonacosane & 0.1046 & 0.2165 & - & - & 0.0359 & 0.0317 \\
\hline Pentacosanoic acid & - & - & 0.0116 & 0.0109 & - & - \\
\hline 1-Hexacosanol & - & - & - & - & 0.0096 & 0.0474 \\
\hline$y$-Tocopherol & - & - & - & - & $\operatorname{tr}$ & - \\
\hline 22-Hydroxydocosanoic acid & - & - & 0.0164 & 0.0133 & - & 0.4934 \\
\hline Hexacosanoic acid & 0.0504 & 0.0420 & 0.0972 & 0.0809 & 0.2351 & 0.1232 \\
\hline Hentriacontene & 1.9274 & 2.5411 & 0.0120 & 0.0379 & - & - \\
\hline Heptacosan-2-ol & - & - & 0.0179 & - & - & - \\
\hline Hentriacontriene & 0.2490 & 0.2911 & - & - & - & - \\
\hline 23-Hy droxytetracosanoic acid & - & - & 0.0653 & 0.0594 & - & - \\
\hline 24-Hy droxytetracosanoic acid & - & - & 0.0044 & 0.0035 & - & - \\
\hline 1-Octacosanol & - & - & - & - & - & 0.1190 \\
\hline$\alpha$-Tocopherol & $\operatorname{tr}$ & $\operatorname{tr}$ & - & - & 0.0071 & 0.0237 \\
\hline$\Delta^{5,3}$-Colestadienol & - & - & - & - & 0.3727 & 0.6061 \\
\hline Brassicasterol & - & - & - & - & 0.1016 & 0.2444 \\
\hline Campesterol & 0.2227 & 0.2788 & - & - & - & - \\
\hline Octacosenoic acid & - & - & 0.0452 & 0.0432 & - & - \\
\hline Octacosanoic acid & 0.0203 & 0.0532 & 0.0132 & 0.0200 & - & - \\
\hline Stigmasterol & 0.0778 & 0.0960 & - & - & - & - \\
\hline Tritriacontene & 0.8440 & 1.0723 & - & - & - & - \\
\hline$\beta$-Sitosterol & 0.0770 & 0.1051 & 0.0968 & 0.1083 & 0.1279 & 0.3656 \\
\hline 25-hydroxy-hexacosanoic acid & - & - & $\operatorname{tr}$ & $\operatorname{tr}$ & - & - \\
\hline Sitostanol & 0.2260 & 0.2961 & - & - & - & - \\
\hline Spinasterol & - & - & - & - & 0.0846 & 0.1036 \\
\hline 1-Triacontanol & - & - & - & - & - & 0.1294 \\
\hline 2-Triacontanol & - & - & 0.0443 & 0.0442 & - & - \\
\hline$\alpha$-Amirin & - & - & - & - & 0.0215 & 0.0432 \\
\hline Cycloartenol & - & - & - & - & 0.0311 & 0.1390 \\
\hline Triacontenoic acid & 0.0575 & $\operatorname{tr}$ & 0.0422 & 0.0290 & - & - \\
\hline Z-24-Tritriac onten-2-one & - & - & 0.0060 & - & - & - \\
\hline 2-Tritriacontenol & - & - & 0.0436 & 0.0384 & - & - \\
\hline Dotriacontenol & 0.0339 & 0.2593 & - & - & - & - \\
\hline Tritriacontenoic acid & - & - & 0.0161 & 0.0124 & - & - \\
\hline Tetratriacontenol & 0.0260 & 0.1654 & 0.0873 & 0.0737 & - & - \\
\hline 2-Tetratriacontanol & - & - & 0.2189 & 0.1791 & - & - \\
\hline Total identified & 11.74 & 20.86 & 2.14 & 3.7 & 5.51 & 12.87 \\
\hline Unidentified & 1.61 & 1.22 & 1.13 & 1.21 & 2.94 & 7.94 \\
\hline Total & 13.35 & 22.08 & 3.27 & 4.91 & 8.45 & 20.815 \\
\hline
\end{tabular}

in sterols and alcohols (Fig. 2) is due to the fact that a fraction of these components is present in the extracts in the esterified forms.

Alkanes and alkenes were also found in the three extracts, coming probably from plants epicuticular waxes.

The pollen from C. ladanifer is the one with higher contents of lipophilic components, followed by Rubus sp. and C. sativa pollen (Fig. 1 and 2).

As far as the individual composition is concerned, C. ladanifer extract is characterized by its high content of fatty acids (before and after alkaline hydrolysis). Among unsaturated fatty acids, linoleic, linolenic, oleic and docosenoic acids are the most abundant. Among saturated fatty acids, palmitic 


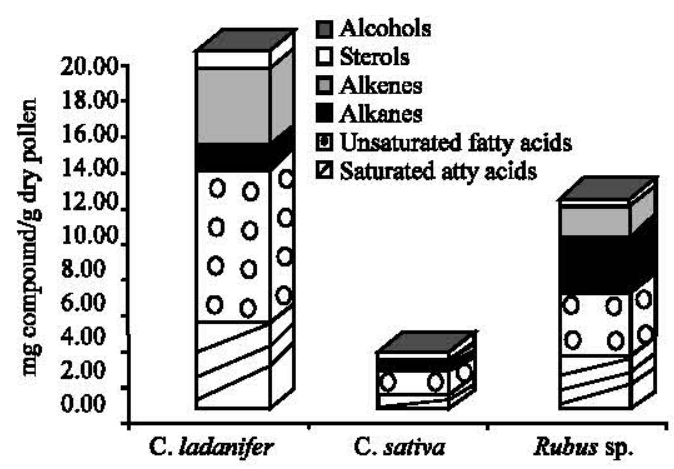

Fig. 2: Major families of compounds, identified in the $n$-hexane extracts of bee pollen, after alkaline hydrolysis

acid is the most abundant, which is in agreement with previous studies (Bonvehí and Jordà, 1997; Saa-Otero et al., 2000). Significant amounts of decanoic and dodecanoic acids were also found. Octanoic, nonanoic and decanedioic acids were found as well, but in lower quantities. Although undecanoic acid exists in small amount, its presence is important, since besides not existing in the other studied species, it is referenced as being an antimicrobial agent (Hornitzky, 2003). Finally, 2-hydroxyocta-9,12-dienoic acid was identified for the first time in the pollen of C. sativa.

Campesterol and sitostanol are the most abundant sterols in C. ladanifer extract, followed by smaller amounts of stigmasterol and $\beta$-sitosterol. $\alpha$-Tocopherol was also identified in the C. ladanifer extract.

In the C. sativa pollen extract the major components are also fatty acids. Oleic acid, followed by linoleic acid, are the most abundant unsaturated fatty acids.

It is worth mentioning that linolenic acid is not present in this pollen extract whereas it is present in considerable amounts in the other two species pollen extracts. This observation is rather important, as it might point out to a specific characteristic of C. sativa pollen from the studied region. In fact, in previous studies on this same species from other geographic locations, linolenic acid is referenced as being one of the most abundant unsaturated fatty acids (Bonvehí and Jordà, 1997; Saa-Otero et al., 2000).

Nonanoic acid is the most abundant saturated fatty acid of $C$. sativa pollen, unlike the preceding species, in which palmitic acid is the most abundant.

Several $\omega$-hydroxyacids, namely 23-hydroxytetracosanoic acid, 24-hydroxytetracosanoic acid and 25-hydroxytetracosanoic acid were, to our knowledge, identified for the first time.

In the C. sativa pollen extract, long-chain alcohols and mainly 1-docosanol, 2-tetratriacontanol and 2-tritiacontenol represent a significant fraction, particularly after alkaline hydrolysis. Oleic acid is the most abundant unsaturated fatty acid in Rubus sp. pollen extract, followed by linolenic and linoleic acids whereas docosanoic acid is the most abundant saturated fatty acid, followed by palmitic acid.

$\beta$-sitosterol and brassicasterol are the most abundant components of Rubus sp. pollen extracts. It is worth mentioning that brassicasterol, lanost-8-en-3-one, cycloartenol, $\beta$-amyrin, stigmasta-3, 5-dien-7-one, $\Delta^{5,7}$-colestadienol and spinasterol were, to our knowledge, identified for the first time in the Rubus sp. pollen.

$\alpha$-Tocopherol and $\gamma$-tocopherol were also identified in Rubus sp. pollen extracts. Since this type of compounds is only found in very small quantities in food and because of its well-known importance, the presence of these vitamins in bee pollen - a product whose potentiality as a dietary supplement is fairly significant-becomes particularly interesting. 
When comparing the studied samples (Fig. 1 and 2), it is obvious that the extract of C. ladanifer is the richest in lipophilic compounds, especially in unsaturated fatty acids. This makes this species particularly interesting from the biological activity point of view, since these acids prevent platelet aggregation (Erasmus, 1996), contributing to the anti-atherosclerotic activity of bee pollen.

On the other hand, the presence of phytosterols makes bee pollen even more interesting, since these compounds are being increasingly used as phytonutrients due to its ability to reduce the levels of blood cholesterol (LDL-C) (Tapiero et al., 2003).

\section{Antimicrobial Activity Essays of Bee Pollen Extracts}

To evaluate the antimicrobial activity of the studied extracts, they were tested towards two Gram negative bacteria-Escherichia coli (CECT 101) and Pseudomonas aeruginosa (CECT 108)-and three Gram positive bacteria-Bacillus cereus (CECT 148), Bacillus subtilis (CECT 498) and Paenibacillus larvae-the latter as an example of causal agent of bees' diseases (American foulbrood).

The results shown refer to the utilization of $300 \mu \mathrm{g}$ of linoleic acid + linolenic acid. We have weighed the adequate amount of extract of each species necessary to obtain an amount of the two acids of $300 \mu \mathrm{g}$, since in the analysed samples these acids represent the major fraction.

The lipophilic extract of $C$. ladanifer pollen has shown the most pronounced inhibitory effect in what concerns the growth of $P$. larvae species, followed by the lipophilic extract of $C$. sativa pollen and then by the extract of Rubus sp. pollen (Fig. 3).

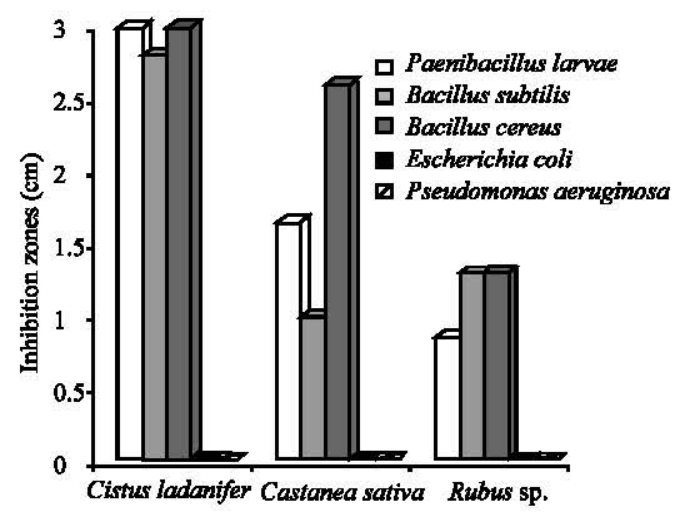

Fig. 3: Inhibition zones resulting from the utilization of the Cistus ladanifer, Castanea sativa and Rubus sp. pollen lipophilic extracts

These results are entirely supported by the quantitative analysis of the extracts, since the extract of C. ladanifer pollen contains the higher content of linoleic acid, besides containing also considerable amounts of other acids (e.g., undecanoic acid) referenced as antimicrobial agents.

Regarding the two other species (C. sativa and Rubus sp.), the high inhibition caused by the extract of C. sativa pollen might have been due to linoleic acid which, in this species' pollen, appears in significant amounts, while in Rubus sp. pollen the $300 \mu \mathrm{g}$ of the sum of the two acids refers almost exclusively to linolenic acid.

The behaviour of the other bacteria studied was similar, since the extract of $C$. ladanifer has shown the higher activity, depending on the tested bacteria. In the case of $E$. coli and $P$. aeruginosa there was no growth inhibition, which might be related to the mechanism of action of the fatty acid versus the mechanism of defence of the Gram negative bacteria.

Given the high antimicrobial activity of the C. ladanifer extract, we might be in the presence of a biocide species for some bacteria, namely Paenibacillus larvae, Bacillus subtilis and Bacillus cereus. 
Therefore, there might be a relationship between the existence of C. ladanifer in the region and the low incidence of American foulbrood. This observation suggests that the possible use of fatty acids as a control strategy for bees' diseases should be better studied given that, unlike antibiotics, no unwanted secondary effects are known. Thus, based on the described results, two strategies can be initially adopted to prevent beehives diseases: i) to displace affected beehives to locations where the native flora produces pollen rich in the fatty acids that have demonstrated significant antimicrobial activity; or ii) to feed the beehives with pollens that possess high concentration of those fatty acids.

\section{Conclusions}

The lipophilic fraction of the three pollen extracts studied were found to be composed essentially by fatty acids, sterols, long chain aliphatic alcohols and also smaller amounts of alkanes and alkenes.

Beyond well known pollen compounds, thirteen new compounds were detected for the fist time in the analysed samples, namely 2-hydroxyocta-9,12-dienoic acid in the C. ladanifer extract, 23-hydroxytetracosanoic acid, 24-hydroxytetracosanoic acid, 25-hydroxytetracosanoic acid and Z-24-tritriaconten-7-one in the C. sativa extract and Z-9-octadecen-1-ol, brassicasterol, lanost-8-en3 -one, cycloartenol, $\beta$-amyrin, stigmasta-3,5-dien-7-one, $\Delta^{5,7}$-colestadienol and spinasterol in the Rubus sp. extract.

The quantitative and qualitative differences found between the composition of the studied samples and published results are probably due to the geographic location allowing a clear distinction in the lipidic profile from region to region. Therefore, we pursue the chemical factors, present in monofloral samples, that discriminate accurately authentic pollen from certain region.

The biological activity results demonstrate an inhibitory action of the studied extracts, particularly of the C. ladanifer extract in the Gram positive bacteria's growth, namely in Paenibacillus larvae, Bacillus subtilis and Bacillus cereus. This might be an important contribution to reduce the risk, or even immunise beehives, to the American foulbrood disease.

\section{Acknowledgements}

Thanks are due to Casa do Mel from Montesinho Natural Park for the collaboration in the pollen sampling in their beehives, to the Aveiro University and to the Agro project 242 ESA-IPB.

\section{References}

Askupmar, E., 1967. Prostatitis and its treatment. Acta Med. Scand., 181: 355-357.

Bianchi, G., C. Murelli and E. Ottaviano, 1990. Maize pollen lipids. Phytochemistry, 29: 739-744.

Bonvehí, J.S. and R.E. Jordà, 1997. Nutrient composition and microbiological quality of honeybee collected pollen in Spain. J. Agric. Food Chem., 45: 725-732.

Boye, N.P., O.P. Salo, N. Hyldebrandt, J.A. Wihl, A. Beran, R.I. Harris and J.R. Lovely, 1990. Immunotherapy of tree pollen allergy with a modified alginate conjugated birch pollen extract compared to an aluminum adsorbed extract. Allergy, 45: 241-248.

Cobo, A., 1984. Polen:problems and perspectives (in spanish in the original: El polen: Problemática y perspectivas). El Campo, pp: 69-77.

Denis, L.J., 1966. Chronic Prostatitis. Acta Urol. Belg., 34: 49-56.

Erasmus, U., 1996. Fats that Heal, Fats that Kill-the Complete Guide to Fats, Oils, Cholesterol and Human Health. Vancouver: Alive Books.

Erdtman, G., 1969. Handbook of Palynology, Morphology, Taxonomy, Ecology-an Introduction to the Study of Pollen and Spores. Munksgaard, Copenhagen. 
Feldlaufer, M.F., W.R. Lusby, D.A. Knox and H. Shimanuki, 1993a. Isolation and identification of linoleic acid as an antimicrobial agent from chalkbrood fungus Ascosphaera apis. Apidologie, 24: 89-94.

Feldlaufer, M.F., D.A. Knox, W.R. Lusby and H. Shimanuki, 1993b. Antimicrobial activity of fatty acids against Bacillus larvae, the causative agent of American foulbrood disease. Apidologie, 24: 95-99.

Freire, C.S.R., A.J.D. Silvestre and C.P. Neto, 2002. Identification of new hydroxy fatty acids and ferulic acid esters in the wood of Eucalyptus globulus. Holzforschung, 56: 143-149.

Hornitzky, M., 2003. Fatty acids, an alternative control strategy for honeybee diseases. Rural Industries Research Development Corporation, Publ No. 03/028, Kingston.

Ji, Z., Z. Caiying, X. Demin, H. Guiqiu, X. Yelu, W. Zhenyi, F. Shengdin, C. Yan and G. Yunlong, 1989. Further study of Pollen Typhae's effects on the production of $\mathrm{PAA}$ and $\mathrm{PGI}_{2}$ by cultured endothelial cells. Thromb. Res., 56: 677-685.

Juzwiak, S., T. Rainska, T. Dutkiewicz, U. Cioch, B. Olenderek, B. Krasowska, L. Rozewicka, Z. Juzyszyn, J. Wojcicki and L. Samochowiec, 1992. Pollen extracts reduce the hepatotoxicity of paracetamol in mice. Phytother. Res., 6: 141-145.

Kosmider, K., J. Wójcicki, L. Samochowiec, M. Woyke and W. Gornik, 1983. Effect of cernilton on platelet aggregation in vivo. Herba Polon., 29: 237-243.

Krell, R., 1996. Vallue-added products from beekeeping. FAO Agricultural Services Bulletin No. 124, Rome.

Liebelt, R.A., 1994. Bee pollen, a wonderful food and a lot more. Am. Bee. J., 134: 611-614.

Manning, R., 2001. Pollen analysis of eucalypts in Western Australia. Rural Industries Research Development Corporation, Publ. No. 01/53, Kingston.

Petschow, B.W., R.P. Batema and L.L. Ford, 1996. Susceptibility of Helicobacter pylori to bactericidal properties of medium-chain monoglycerides and free fatty acids. Antimicrob. Ag. Chemother., 40: 302-306.

Rugendorff, E.W., W. Weidner, L. Ebeling and A.C. Buck, 1993. Results of treatment with pollen extract (Cernilton N) in chronic prostatitis and prostatodynia. Brit. J. Urol., 71: 433-438.

Saa-Otero, M.P., E. Díaz-Losada and E. Fernández-Gómez, 2000. Analysis of fatty acids, proteins and ethereal extract in honeybee pollen. Considerations of their floral origin. Grana, 39: 175-181.

Samochowiec, L., T. Dutkiewicz, J. Wójcicki and J. Gieldanowski, 1992. The influence of pollen extracts (Cernitin GBX and Cernitin T60) on Allergic Reactions. Phytother. Res., 6: 314-317.

Staff, I.A., P.E. Taylor, P. Smith, M.B. Sing and R.B. Knox, 1990. Cellular localization of water soluble allergenic proteins in rye-grass (Lolium perenne L.) pollen using monoclonal and specific IgE antibodies with immunogold probes. Histochem. J., 22: 276-290.

Tapiero, H., D.M. Townsend and K.W. Tew, 2003. Phytosterols in the prevention of human pathologies. Biomed. Pharmacother., 57: 321-325.

Wenning, C.J., 2003. Pollen and the honey bee. Am. Bee J., 143: 394-397.

Wójcicki, J. and K. Kosmider, L. Samochowiec and M. Woyke, 1983. Clinical evaluation of cernilton as lipid-lowering agent. Herba Polon., 29: 55-63.

Wójcicki, J., L. Samochowiec, 1984. Further studies on cernitins: Screening of the hypolipidemic activity in rats. Herba Polon., 30: 115-121.

Wójcicki , J., L. Samochowiec, B. Bartlomowicz, A. Hinek, M. Jaworska and B. Gawronska-Szklarz, 1986. Effect of pollen extract on the development of experimental atherosclerosis in rabbits. Atherosclerosis, 62: 39-45.

Wortmann, F., 1981. Oral immunotherapy. Clin. Immunol. Allerg, pp: 389-398.

Zhao, J., C.Y. Zhang, D.M. Xu, G.Q. Huang, Y.L. Xu and Z.Y. Wang, 1990. The antiatherogenic effects of components isolated from pollen Typhae. Thromb. Res., 57: 957-966. 